\title{
Guaranteed Cost Finite-Time Control of Discrete-Time Positive Impulsive Switched Systems
}

\author{
Leipo Liu $\left(\mathbb{D}\right.$, Hao Xing, Xiangyang Cao, Zhumu Fu $\mathbb{D}^{D}$, and Shuzhong Song \\ College of Information Engineering, Henan University of Science and Technology, Luoyang 471023, China \\ Correspondence should be addressed to Leipo Liu; liuleipo123@163.com
}

Received 6 July 2017; Revised 22 January 2018; Accepted 12 March 2018; Published 16 April 2018

Academic Editor: Peter Giesl

Copyright (C) 2018 Leipo Liu et al. This is an open access article distributed under the Creative Commons Attribution License, which permits unrestricted use, distribution, and reproduction in any medium, provided the original work is properly cited.

\begin{abstract}
This paper considers the guaranteed cost finite-time boundedness of discrete-time positive impulsive switched systems. Firstly, the definition of guaranteed cost finite-time boundedness is introduced. By using the multiple linear copositive Lyapunov function (MLCLF) and average dwell time (ADT) approach, a state feedback controller is designed and sufficient conditions are obtained to guarantee that the corresponding closed-loop system is guaranteed cost finite-time boundedness (GCFTB). Such conditions can be solved by linear programming. Finally, a numerical example is provided to show the effectiveness of the proposed method.
\end{abstract}

\section{Introduction}

As a special kind of positive systems [1-3], the positive switched systems whose output and state are nonnegative whenever the initial condition and input are nonnegative have been found in many applications such as communication networks [4], viral mutation [5], and formation flying [6]. There have been many available results about continuoustime positive switched systems [7-11] and discrete-time positive switched systems [12-14].

However, most results mentioned above focus on the classical Lyapunov stability, which guarantees the stability in an infinite-time interval. Different from the Lyapunov stability concept, the finite-time stability requires that the states do not exceed a certain bound during a fixed finitetime interval. The paper [15] firstly defined the definition of finite-time stability (FTS) for linear deterministic systems. Recently, [16] firstly extended the concept of FTS to positive switched systems and gave some FTS conditions of positive switched systems. So far, there have been a few meaningful results about FTS of positive switched systems; see [1720]. In these results, to make the best of the nature of positivity, the MLCLF approach has been widely used and became a powerful tool for the analysis and synthesis of positive switched systems. Due to the wide application of digital controllers, some researches have been done on the
FTS of discrete-time positive switched systems. The paper [21] investigated the problem of robust finite-time stability and stabilization of a class of discrete-time positive switched systems. The paper [22] studied the problem of finite-time control of a class of discrete impulsive switched positive timedelay systems under asynchronous switching, but the effect of disturbance was ignored.

Moreover, in most of practical applications, the researchers are more interested in designing the control system which is not only finite-time stable but also guarantees an adequate level of performance. One method to this problem is the so-called guaranteed cost finite-time control. Some remarkable results have been presented; see [23-27]. These results mainly focus on nonpositive systems. Very recently, in [28], guaranteed cost finite-time control was extended to fractional-order positive switched systems and a cost function for fractional-order positive systems (or fractional-order positive switched systems) was proposed. In [29], the problem of guaranteed cost finite-time control for positive switched linear systems with time-varying delays was considered and a cost function of positive systems (or positive switched systems) was also presented. Based on [29], [30] extended guaranteed cost finite-time control to positive switched nonlinear systems with $D$-perturbation. It is worth noting that [28-30] are involved in continuous-time positive switched systems. However, the problem of guaranteed 
cost finite-time control for discrete-time positive impulsive switched systems is still open, which inspires us for this study.

In this paper, we consider the problem of GCFTB of discrete-time positive impulsive switched systems by constructing the MLCLF with average dwell time (ADT) technique. Firstly, the concept of guaranteed cost finite-time boundedness is extended to discrete-time positive impulsive switched systems. Secondly, a state feedback controller is designed and sufficient conditions are obtained to guarantee that the closed-loop system is GCFTB. Some sufficient conditions are obtained by linear programming.

The rest of the paper is organized as follows. Section 2 gives some necessary preliminaries and problem statements. In Section 3, the main results are given. In Section 4, a numerical example is provided. Section 5 concludes the paper.

Notations. The representation $A>0(\geqslant 0, \prec 0, \preccurlyeq 0)$ means that $a_{i j}>0(\geq 0,<0, \leq 0)$, which is also applying to a vector. $A>B(A \geq B)$ means that $A-B>0(A-B \geq 0)$. $R_{+}^{n}$ is the $n$-dimensional nonnegative (positive) vector space. $R^{n \times n}$ denotes the space of $n \times n$ matrices with real entries. $I_{l}$ represents the $l$-dimensional vector $[1, \ldots, 1]^{T} . A^{T}$ denotes the transpose of matrix $A$. 1-norm $\|x\|$ is defined by $\|x\|=$ $\sum_{k=1}^{n}\left|x_{k}\right| . N$ and $N^{+}$are the sets of nonnegative and positive integers. $Z^{+}$denotes the set of positive integers. Matrices are assumed to have compatible dimensions for calculating if their dimensions are not explicitly stated.

\section{Preliminaries and Problem Statements}

Consider the following discrete-time positive impulsive switched systems:

$$
\begin{gathered}
x(k+1)=A_{\sigma(k)} x(k)+B_{\sigma(k)} u(k)+C_{\sigma(k)} w(k), \\
k \neq k_{m}-1, m \in Z^{+} \\
x(k+1)=E_{\sigma(k)} x(k), \quad k=k_{m}-1, m \in Z^{+},
\end{gathered}
$$

where $k \in N, x(t) \in R^{n}$ is the system state, and $u(t) \in R^{l}$ represents the control input. $\sigma(k)$ represents switching signal of system and takes values in a finite set $I=1,2, \ldots, S$, $S \in N^{+}$. In general, $A_{i}, B_{i}, C_{i}$, and $E_{i}$ are the $i$ th subsystem if $\sigma(k)=i \in I . k_{0}=0$ is the initial time. $k_{m}(m \in$ $Z^{+}$) denotes the $m$ th impulsive switching instant. Moreover, $\sigma(k)=i \in I$ means that the $i$ th subsystem is active. $\sigma(k-1)=j$ and $\sigma(k)=i(i \neq j)$ indicate that $k$ is a switching instant at which the system is switched from the $j$ th subsystem to the $i$ th subsystem. At switching instants, there exist impulsive jumps described by (1). $A_{p}, B_{p}, C_{p}$, and $E_{p}$ are constant matrices with suitable dimensions, $w(k) \in R^{l}$ is the exogenous disturbance and defined as

$$
\sum_{k=0}^{T_{f}}\|w(k)\| \leq d,
$$

with a known scalar $d>0$ and a given finite-time threshold value $T_{f}$. (1).

Next, we will give some definitions and lemmas for system

Definition 1. System (1) is said to be positive if for any switching signals $\sigma(k)$, any disturbance input $w(k) \geq 0$, and control input $u(k) \geq 0$, the corresponding trajectory satisfies $x(k) \geq 0$ for all $k \geq 0$.

Lemma 2 (see [25]). System (1) is positive if and only if $A_{i} \geq 0$, $B_{i} \geq 0, C_{i} \geq 0$, and $E_{i} \geq 0$, where $i \in I$.

Definition 3. For any switching signal $\sigma(k)$ and any $t_{2} \geq$ $t_{1} \geq 0$, let $N_{\sigma}\left(t_{1}, t_{2}\right)$ denote the switching numbers over the interval $\left[t_{1}, t_{2}\right)$. For given $t_{l}>0$ and $n_{0}>0$, if the inequality

$$
N_{\sigma}\left(t_{1}, t_{2}\right) \leq n_{0}+\frac{t_{2}-t_{1}}{t_{\iota}}
$$

holds, then $t_{t}$ is called an average dwell time, and $n_{0}$ is called a chattering bound. Generally, we choose $n_{0}=0$.

Definition 4 (finite-time stability (FTS)). For a given time $T_{f}$ and two vectors $\alpha>\beta>0$, discrete-time positive impulsive switched system (1) with $\omega(k) \equiv 0$ is said to be FTS with respect to $\left(\alpha, \beta, T_{f}, \sigma(k)\right)$, if

$$
\begin{aligned}
& x^{T}(0) \beta \leq 1 \Longrightarrow \\
& x^{T}(k) \alpha<1, \quad \forall k \in\left[0, T_{f}\right],
\end{aligned}
$$

where $k$ is an any time point on the time interval $\left[0, T_{f}\right]$.

Definition 5 (finite-time boundedness (FTB)). For a given constant $T_{f}$, and two vectors $\alpha>\beta>0$, discrete-time positive impulsive switched system (1) is said to be FTB with respect to $\left(\alpha, \beta, T_{f}, d, \sigma(k)\right)$, where $w(t)$ satisfies (2), if

$$
\begin{aligned}
& x^{T}(0) \beta \leq 1 \Longrightarrow \\
& x^{T}(k) \alpha<1, \quad \forall k \in\left[0, T_{f}\right],
\end{aligned}
$$

where $k$ is an any time point on the time interval $\left[0, T_{f}\right]$.

Now we give some new definitions for our further study.

Definition 6. Define the cost function of discrete-time positive impulsive switched system (1) as follows:

$$
J=\sum_{s=0}^{T_{f}-1}\left(x^{T}(s) R_{1}+u^{T}(s) R_{2}\right),
$$

where $R_{1}>0$ and $R_{2}>0$ are two given vectors.

Remark 7. It should be noted that the proposed cost function is different from the general one, such as [26-28]; this definition provides a more useful description, because it takes full advantage of the characteristics of nonnegative states of discrete-time positive impulsive switched systems.

Definition 8 (GCFTB). For a given time constant $T_{f}$ and two vectors $\varsigma>\rho>0$, consider discrete-time positive impulsive 
switched system (1) and cost function (6); if there exist a control law $u(t)$ and a positive scalar $J^{\star}$ such that the closedloop system is FTB with respect to $\left(\alpha, \beta, T_{f}, d, \sigma(k)\right)$ and the cost function satisfies $J \leq J^{\star}$, then the closed-loop system is called GCFTB, where $J^{\star}$ is a guaranteed cost value and $u(t)$ is a guaranteed cost finite-time controller.

\section{Main Results}

3.1. Guaranteed Cost Finite-Time Boundedness Analysis. In this subsection, we will focus on the problem of GCFTB for discrete-time positive impulsive switched system (1) with $u(k)=0$. The following theorem gives sufficient conditions of GCFTB for system (1) with $u(k)=0$.

Theorem 9. Consider the discrete-time positive impulsive switched system (1) with $u(k)=0$, for a given time constant $T_{f}$, vectors $\alpha>\beta>0$ and $R_{1}>0$; if there exist a set of positive vectors $v_{i}, v_{j}, i \neq j, i, j \in I$ and positive constants $\phi_{1}, \phi_{2}, \varsigma$, $\xi>1, \mu>1$ such that the following inequalities hold:

$$
\begin{gathered}
A_{i}^{T} \nu_{i}+R_{1}-\xi \nu_{i} \prec 0, \\
C_{i}^{T} \nu_{i}-\varsigma I_{l} \prec 0, \\
E_{i}^{T} \nu_{j}-\mu \nu_{i} \prec 0, \\
\phi_{1} \alpha \prec \nu_{i} \prec \phi_{2} \beta, \\
\phi_{1}>\left(\phi_{2}+\varsigma d\right) \xi^{T_{f}},
\end{gathered}
$$

where $v_{i}=\left[v_{i 1}, v_{i 2}, \ldots, v_{i n}\right]^{T}$ and $v_{i r}$ represents the ith elements of the vectors $v_{i}$, respectively, then under the following ADT scheme:

$$
T_{\alpha}>T_{\alpha}^{*}=\frac{T_{f} \ln \mu}{\ln \left(\phi_{1}\right)-\ln \left(\phi_{2}+\varsigma d\right)-T_{f} \ln (\xi)}
$$

system (1) with $u(k)=0$ is GCFTB with respect to $\left(\alpha, \beta, T_{f}, d, \sigma(k)\right)$ and the guaranteed cost value of system (1) with $u(k)=0$ is given by

$$
J=\sum_{k=0}^{T_{f}-1} x^{T}(k) R_{1} \leq J^{*}=\xi^{T_{f}} \mu^{T_{f} / T_{\alpha}^{*}}\left(\phi_{2}+\varsigma d\right)
$$

Proof. Construct the multiple linear copositive Lyapunov function (MLCLF) for system (1) with $u(k)=0$ as follows:

$$
V_{i}(x(k))=x^{T}(k) v_{(i)}
$$

where $i \in I$.

Suppose a switching sequence $0=k_{0} \leq k_{1} \leq \cdots \leq k_{m} \leq$ $k_{m+1} \leq \cdots \leq T_{f}$. Without loss of generality, we assume that subsystem $i$ is activated at the switching instant $k_{m-1}$ and the subsystem $j$ is activated at the switching instant $k_{m}$.
When $k \in\left[k_{m-1}, k_{m}-1\right), m \in N, \sigma(k)=\sigma(k+1)=i$, along the trajectory of system (1) with $u(k)=0$, the difference of the MLCLF is

$$
\begin{aligned}
\triangle & V_{i}(x(k))+x^{T}(k) R_{1} \\
& =V_{i}(x(k+1))-V_{i}(x(k))+x^{T}(k) R_{1} \\
& =x^{T}(k)\left(A_{i}^{T} v_{i}+R_{1}-v_{i}\right)+\omega^{T}(k) C_{i}^{T} v_{i} .
\end{aligned}
$$

From (7), (8) and $x(k) \geq 0, k \in N$, we have

$$
\begin{aligned}
& \triangle V_{i}(x(k))+x^{T}(k) R_{1} \\
& \quad \leq(\xi-1) x^{T}(k) v_{i}+\varsigma \omega^{T}(k) I_{l} .
\end{aligned}
$$

It implies that

$$
V_{i}(x(k+1)) \leq \xi x^{T}(k) v_{i}+\varsigma\|\omega(k)\|_{1} .
$$

When $k=k_{m}-1, \sigma(k+1)=\sigma\left(k_{m}\right)=j, \sigma(k)=\sigma\left(k_{m}-1\right)=$ $i, i \neq j$. Along the trajectory of system (1) with $u(k)=0$, we have

$$
\begin{aligned}
& V_{j}(x(k+1))-\mu V_{i}(x(k)) \\
& \quad=x^{T}(k+1) v_{j}-\mu x^{T}(k) v_{i} \\
& \quad \leq x^{T}(k)\left(E_{i}^{T} v_{j}-\mu v_{i}\right) .
\end{aligned}
$$

From (9) and $x(k) \geq 0, k \in N$, we have

$$
V_{j}(x(k+1)) \leq \mu V_{i}(x(k)), \quad i \neq j .
$$

So, when $k \in\left[k_{m}, k_{m+1}\right)$, from (19), we get

$$
\begin{aligned}
& V_{\sigma(k)}(x(k))< \xi^{k-k_{m}} V_{\sigma\left(k_{m}\right)}\left(x\left(k_{m}\right)\right) \\
&+\varsigma \sum_{s=k_{m}}^{k-1} \xi^{k-1-s}\|\omega(s)\|_{1} \\
&< \mu \xi^{k-k_{m}} V_{\sigma\left(k_{m}-1\right)}\left(x\left(k_{m}-1\right)\right) \\
&+\varsigma \sum_{s=k_{m}}^{k-1} \xi^{k-1-s}\|\omega(s)\|_{1} .
\end{aligned}
$$


Repeating the procedure of (20) and noting $\xi>1$, we obtain

$$
\begin{aligned}
V_{\sigma(k)}(x(k))< & \xi^{k-k_{m}} V_{\sigma\left(k_{m}\right)}\left(x\left(k_{m}\right)\right) \\
& +\varsigma \sum_{s=k_{m}}^{k-1} \xi^{k-1-s}\|\omega(s)\|_{1} \\
< & \mu \xi^{k-k_{m}} V_{\sigma\left(k_{m}-1\right)}\left(x\left(k_{m}-1\right)\right) \\
& +\varsigma \sum_{s=k_{m}}^{k-1} \xi^{k-1-s}\|\omega(s)\|_{1} \\
< & \mu \xi^{k-k_{m}} \xi^{k_{m}-1-k_{m-1}} V_{\sigma\left(k_{m-1}\right)}\left(x\left(k_{m-1}\right)\right) \\
& +\varsigma \sum_{s=k_{m}}^{k-1} \xi^{k-1-s}\|\omega(s)\|_{1} \\
& +\mu \varsigma \sum_{s=k_{m-1}}^{k_{m}-1} \xi^{k_{m}-1-s}\|\omega(s)\|_{1} \\
& +\mu \xi^{k-k_{m-1}} V_{\sigma\left(k_{m-1}\right)}\left(x\left(k_{m-1}\right)\right) \\
& +\varsigma \sum_{s=k_{m}}^{k-1} \xi^{k-1-s} \| \omega(s) \eta_{1} \\
& \xi^{k_{m}-1-s}\|\omega(s)\|_{1} . \\
& \\
& \\
& \\
& \\
&
\end{aligned}
$$

By iterative operation, we get

$$
\begin{aligned}
V_{\sigma(k)}(x(k))< & \mu^{2} \xi^{k-k_{m-2}} V_{\sigma\left(k_{m-2}\right)}\left(x\left(k_{m-2}\right)\right) \\
& +\varsigma \sum_{s=k_{m}}^{k-1} \xi^{k-1-s}\|\omega(s)\|_{1} \\
& +\mu \varsigma \sum_{s=k_{m-1}}^{k_{m}-1} \xi^{k_{m}-1-s}\|\omega(s)\|_{1} \\
& +\mu^{2} \varsigma \sum_{s=k_{m-2}}^{k_{m-1}-1} \xi^{k_{m}-1-s}\|\omega(s)\|_{1} \leq \cdots \\
\leq & \mu^{N_{\sigma}\left(k, k_{0}\right)} \xi^{k-k_{0}} V_{\sigma\left(k_{0}\right)}\left(x\left(k_{0}\right)\right) \\
& +\varsigma \sum_{s=k_{m}}^{k-1} \xi^{k-1-s}\|\omega(k)\|_{1} \\
& +\mu \varsigma \sum_{s=k_{m-1}}^{k_{m}-1} \xi^{k_{m}-1-s}\|\omega(s)\|_{1}
\end{aligned}
$$

$$
\begin{gathered}
+\mu^{2} \varsigma \sum_{s=k_{m-2}}^{k_{m-1}-1} \xi^{k_{m}-1-s}\|\omega(s)\|_{1}+\cdots \\
+\mu^{N_{\sigma}\left(k-1, k_{0}\right)} \varsigma \sum_{s=k_{0}}^{k_{1}-1} \xi^{k_{1}-1-s}\|\omega(s)\|_{1} \\
=\mu^{N_{\sigma}\left(k, k_{0}\right)} \xi^{k-k_{0}} V_{\sigma\left(k_{0}\right)}\left(x\left(k_{0}\right)\right) \\
+\mu^{N_{\sigma}(k-1, s)} \varsigma \sum_{s=k_{0}}^{k-1} \xi^{k-1-s}\|\omega(s)\|_{1} .
\end{gathered}
$$

According to (2), $\xi>1$ and $\mu>1$, we also have

$$
V_{\sigma(k)}(x(k))<\mu^{N_{\sigma}\left(k, k_{0}\right)} \xi^{k-k_{0}}\left(V_{\sigma\left(k_{0}\right)}\left(x\left(k_{0}\right)\right)+\varsigma d\right) .
$$

From (10) and (14), we have

$$
\begin{gathered}
V_{\sigma(k)}(x(k))=x^{T}(k) \nu_{\sigma(k)} \geq \phi_{1} x^{T}(k) \alpha, \\
V_{\sigma\left(k_{0}\right)}\left(x\left(k_{0}\right)\right)=x^{T}(0) \nu_{\sigma\left(k_{0}\right)} \leq \phi_{2} x^{T}(0) \beta .
\end{gathered}
$$

From (23) to (24) and $k \in\left[0, T_{f}\right]$, we obtain

$$
\begin{aligned}
x^{T}(k) \alpha & \leq \frac{1}{\phi_{1}} \mu^{T_{f} / T_{\alpha}} \xi^{T_{f}}\left(\phi_{2} x^{T}(0) \beta+\varsigma d\right) \\
& \leq \frac{1}{\phi_{1}} \mu^{T_{f} / T_{\alpha}} \xi^{T_{f}}\left(\phi_{2}+\varsigma d\right) .
\end{aligned}
$$

Substituting (12) into (25), one has

$$
x^{T}(k) \alpha<1, \quad k \in\left[0, T_{f}\right] .
$$

According to Definition 5, we conclude that system (1) with $u(k)=0$ is FTB with respect to $\left(\alpha, \beta, T_{f}, d, \sigma(k)\right)$.

Next, we will give the guaranteed cost value of system (1) with $u(k)=0$. know

When $k \in\left[k_{m-1}, k_{m}-1\right), m \in N$, according to (16), we

$$
V_{i}(x(k)) \leq \xi x^{T}(k) v_{i}+\varsigma\|\omega(k)\|_{1}-x^{T}(k) R_{1} .
$$

Similar to the proof process of (17)-(22), for any $k \in\left[0, T_{f}\right]$ and $\mu>1$, we can obtain

$$
\begin{aligned}
V_{\sigma(k)}(x(k))< & \mu^{N_{\sigma}\left(k, k_{0}\right)} \xi^{k-k_{0}} V_{\sigma\left(k_{0}\right)}\left(x\left(k_{0}\right)\right) \\
& +\mu^{N_{\sigma}\left(k, k_{0}\right)} \varsigma \sum_{s=0}^{k-1} \xi^{k-s}\|\omega(s)\|_{1} \\
& -\sum_{s=k_{0}}^{k-1} x^{T}(s) R_{1} .
\end{aligned}
$$

Noting that $V_{\sigma(k)}(x(k))>0,(28)$ can be rewritten as

$$
\begin{aligned}
0< & \mu^{N_{\sigma}\left(k, k_{0}\right)} \xi^{k-k_{0}} V_{\sigma\left(k_{0}\right)}\left(x\left(k_{0}\right)\right) \\
& +\mu^{N_{\sigma}\left(k, k_{0}\right)} \varsigma \sum_{s=0}^{k-1} \xi^{k-s}\|\omega(s)\|_{1}-\sum_{s=0}^{k-1} x(s)^{T} R_{1} .
\end{aligned}
$$


Substituting (2) into (29) and letting $k=T_{f}$, we get

$$
\begin{gathered}
\sum_{s=0}^{T_{f}-1} x(s)^{T} R_{1}<\mu^{N_{\sigma}\left(k, k_{0}\right)} \xi^{k-k_{0}}\left(V_{\sigma\left(k_{0}\right)}\left(x\left(k_{0}\right)\right)+\varsigma d\right) \\
<\mu^{N_{\sigma}\left(T_{f}, k_{0}\right)} \xi^{T_{f}-k_{0}}\left(V_{\sigma\left(k_{0}\right)}\left(x\left(k_{0}\right)\right)+\varsigma d\right) .
\end{gathered}
$$

Then we can obtain

$$
J=\sum_{s=0}^{T_{f}-1} x^{T}(s) R_{1} \leq J^{*}=\xi^{T_{f}} \mu^{T_{f} / T_{\alpha}^{*}}\left(\phi_{2}+\varsigma d\right) .
$$

Therefore, according to Definition 8, we can conclude that system (1) with $u(k)=0$ is GCGTB. Thus, the proof is completed.

3.2. Guaranteed Cost Finite-Time Controller Design. In this subsection, we are concerned with the guaranteed cost finitetime controller design of discrete-time positive impulsive switched system (1). Under the controller $u(t)=K_{\sigma(k)} x(k)$, the corresponding closed-loop system is given by

$$
\begin{gathered}
x(k+1)=\left(A_{\sigma(k)}+B_{\sigma(k)} K_{\sigma(k)}\right) x(k)+C_{\sigma(k)} w(k), \\
k \neq k_{m}-1, m \in Z^{+}, \\
x(k+1)=E_{\sigma(k)} x(k), \quad k=k_{m}-1, m \in Z^{+} .
\end{gathered}
$$

By Lemma 2, to guarantee the positivity of system (32), $A_{i}+$ $B_{i} K_{i} \geq 0$ should be satisfied, $\forall i \in I$. The following Theorem 10 gives some sufficient conditions to guarantee that the closedloop system (32) is GCFTB.

Theorem 10. Consider the discrete-time positive impulsive switched system (32), for a given time constant $T_{f}$, vectors $\alpha>\beta>0, R_{1}>0$, and $R_{2}>0$; if there exist positive vectors $v_{i}, f_{i}, i \in I$ and positive constants $\phi_{1}, \phi_{2}, \varsigma, \xi>1, \mu>1$, such that ((8)-(11)) and the following inequalities hold:

$$
\begin{array}{r}
A_{i}+B_{i} K_{i} \geq 0, \\
A_{i}^{T} \nu_{i}+f_{i}+R_{1}+K_{i}^{T} R_{2}-\xi \nu_{i} \prec 0,
\end{array}
$$

where $f_{i}=K_{i}^{T} B_{i}^{T} v_{i}, v_{i}=\left[v_{i 1}, v_{i 2}, \ldots, v_{i n}\right]^{T}$, and $v_{i r}$ represents the ith elements of the vectors $v_{i}$, then under the following ADT scheme (12), the resulting closed-loop system (32) is GCFTB with respect to $\left(\alpha, \beta, T_{f}, d, \sigma(k)\right)$ and the guaranteed cost value of system (32) is given by

$$
\begin{aligned}
J & =\sum_{k=0}^{T_{f}-1}\left(x^{T}(k) R_{1}+x^{T}(k) K_{i}^{T} R_{2}\right) \leq J^{*} \\
& =\xi^{T_{f}} \mu^{T_{f} / T_{\alpha}^{*}}\left(\phi_{2}+\varsigma d\right) .
\end{aligned}
$$

Proof. From (33), we know that $A_{i}+B_{i} K_{i} \geq 0$. According to Lemma 2, system (32) is positive. Next, we prove the guaranteed cost finite-time stability of system (32).
Replacing $R_{1}$ in (27) with $R_{1}+K_{i}^{T} R_{2}$, we have

$$
\begin{aligned}
V_{i}(x(k)) \leq & \xi x^{T}(k) v_{i}+\varsigma\|\omega(k)\|_{1} \\
& -x^{T}(k)\left(R_{1}+K_{i}^{T} R_{2}\right) .
\end{aligned}
$$

Similar to the proof process of (17)-(22), for any $k \in\left[0, T_{f}\right]$ and $\mu>1$, we can obtain

$$
\begin{aligned}
V_{\sigma(k)}(x(k))< & \mu^{N_{\sigma}\left(k, k_{0}\right)} \xi^{k-k_{0}} V_{\sigma\left(k_{0}\right)}\left(x\left(k_{0}\right)\right) \\
& +\mu^{N_{\sigma}\left(k, k_{0}\right)} \varsigma \sum_{s=0}^{k-1} \xi^{k-s}\|\omega(s)\|_{1} \\
& -\sum_{s=k_{0}}^{k-1} x^{T}(s)\left(R_{1}+K_{i}^{T} R_{2}\right) .
\end{aligned}
$$

Noting that $V_{\sigma(k)}(x(k))>0,(37)$ can be rewritten as

$$
\begin{array}{r}
0<\mu^{N_{\sigma}\left(k, k_{0}\right)} \xi^{k-k_{0}} V_{\sigma\left(k_{0}\right)}\left(x\left(k_{0}\right)\right) \\
+\mu^{N_{\sigma}\left(k, k_{0}\right)} \varsigma \sum_{s=0}^{k-1} \xi^{k-s}\|\omega(k)\|_{1} \\
-\sum_{s=0}^{k-1} x(s)^{T}\left(R_{1}+K_{i}^{T} R_{2}\right) .
\end{array}
$$

Substituting (2) into (38) and letting $k=T_{f}$, we get

$$
\begin{aligned}
& \sum_{s=0}^{T_{f}-1} x(s)^{T}\left(R_{1}+K_{i}^{T} R_{2}\right) \\
& <\mu^{N_{\sigma}\left(k, k_{0}\right)} \xi^{k-k_{0}}\left(V_{\sigma\left(k_{0}\right)}\left(x\left(k_{0}\right)\right)+\varsigma d\right) \\
& <\mu^{N_{\sigma}\left(T_{f}, k_{0}\right)} \xi^{T_{f}-k_{0}}\left(V_{\sigma\left(k_{0}\right)}\left(x\left(k_{0}\right)\right)+\varsigma d\right) .
\end{aligned}
$$

From (12), we can obtain

$$
\begin{aligned}
J & =\sum_{s=0}^{T_{f}-1} x^{T}(s)\left(R_{1}+K_{i}^{T} R_{2}\right) \leq J^{*} \\
& =\xi^{T_{f}} \mu^{T_{f} / T_{\alpha}^{*}}\left(\phi_{2}+\varsigma d\right) .
\end{aligned}
$$

The proof is completed.

Next, an algorithm is presented to obtain the feedback gain matrices $K_{i}, i \in I$.

\section{Algorithm 11.}

Step 1. Inputting matrices $A_{i}, B_{i}, C_{i}, E_{i}, R_{1}, R_{2}, \alpha$, and $\beta$.

Step 2. By adjusting the parameters $\xi$, $\mu$, then solving (8)-(11) and (34) via linear programming, positive vectors $v_{p}, f_{p}$, and $K_{p}$ can be obtained. If $K_{p}>0$, turn to the next step. Otherwise, return to Step 1.

Step 3. Substituting $\nu_{p}$ and $K_{p}$ into $f_{p}=K_{p}^{T} B_{p}^{T} \nu_{p}, \tilde{f}_{p}$ can be obtained. If $\tilde{f}_{p}-f_{p} \preceq 0$, then $K_{p}$ are admissible. Otherwise, return to Step 1 . 


\section{Numerical Example}

Consider the discrete-time positive impulsive switched system (1) with the parameters as follows:

$$
\begin{aligned}
& A_{1}=\left[\begin{array}{ll}
0.3 & 0.2 \\
0.2 & 0.3
\end{array}\right] \text {, } \\
& B_{1}=\left[\begin{array}{ll}
0.2 & 0.1 \\
0.3 & 0.1
\end{array}\right] \text {, } \\
& C_{1}=\left[\begin{array}{ll}
0.3 & 0.1 \\
0.2 & 0.3
\end{array}\right] \text {, } \\
& E_{1}=\left[\begin{array}{cc}
0.3 & 0 \\
0 & 0.3
\end{array}\right] \text {, } \\
& A_{2}=\left[\begin{array}{ll}
0.4 & 0.2 \\
0.3 & 0.2
\end{array}\right] \text {, } \\
& B_{2}=\left[\begin{array}{ll}
0.3 & 0.1 \\
0.1 & 0.2
\end{array}\right] \text {, } \\
& C_{2}=\left[\begin{array}{ll}
0.2 & 0.3 \\
0.1 & 0.2
\end{array}\right], \\
& E_{2}=\left[\begin{array}{cc}
0.3 & 0 \\
0 & 0.3
\end{array}\right] \text {, } \\
& R_{1}=\left[\begin{array}{l}
0.1 \\
0.1
\end{array}\right] \text {, } \\
& R_{2}=\left[\begin{array}{l}
0.3 \\
0.1
\end{array}\right] \text {, } \\
& \alpha=\left[\begin{array}{l}
0.02 \\
0.03
\end{array}\right] \text {, } \\
& \beta=\left[\begin{array}{l}
0.1 \\
0.2
\end{array}\right] \text {. }
\end{aligned}
$$

Choosing $T_{f}=5, \xi=1.1, \mu=1.05, w(k)=$ $\left[0.1(\cos (0.3 k))^{2}, 0.1(\cos (0.3 k))^{2}\right]^{T}, d=1.2$. Solving the inequalities in Theorem 10 by linear programming, we have

$$
\begin{aligned}
& v_{1}=\left[\begin{array}{l}
0.1332 \\
0.2021
\end{array}\right], \\
& v_{2}=\left[\begin{array}{l}
0.1332 \\
0.2023
\end{array}\right], \\
& f_{1}=\left[\begin{array}{l}
0.1263 \\
0.1432
\end{array}\right],
\end{aligned}
$$

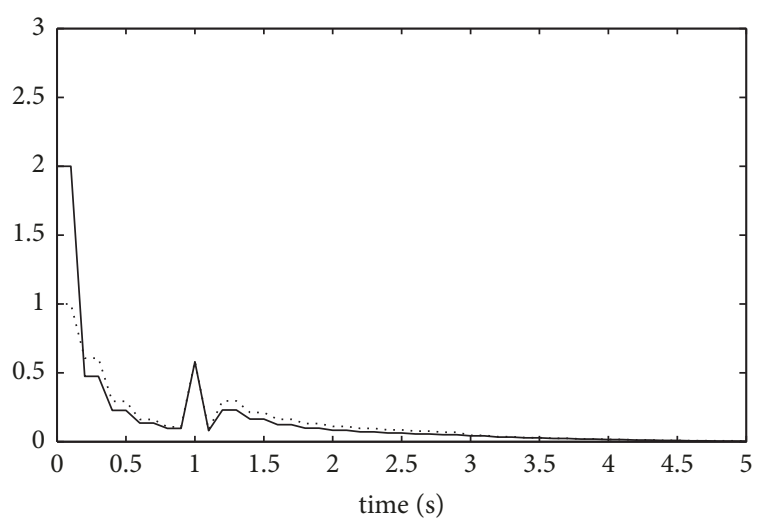

$\cdots \cdots \times 1$

$-x 2$

Figure 1: State trajectories of closed-loop system (1).

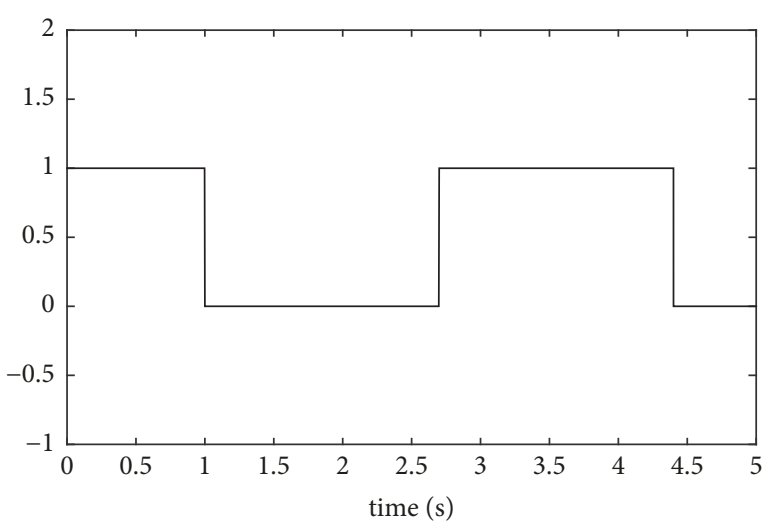

FIGURE 2: Switching signal of system (1) with ADT.

$$
\begin{aligned}
& f_{2}=\left[\begin{array}{l}
0.1113 \\
0.1328
\end{array}\right], \\
& K_{1}=\left[\begin{array}{ll}
0.0246 & 0.0252 \\
0.0251 & 0.0254
\end{array}\right], \\
& K_{2}=\left[\begin{array}{ll}
0.0242 & 0.0251 \\
0.0247 & 0.0253
\end{array}\right] .
\end{aligned}
$$

It is easy to confirm that $\tilde{f}_{p}-f_{p} \leq 0$ and (33) is satisfied; then $K_{p}$ are admissible. According to (12), we get $T_{\alpha}^{*}=1.7$.

The simulation results are shown in Figures 1-3, where the initial conditions of system $(1)$ are $x(0)=[1,2]^{T}$, which meet the condition $x^{T}(k) \alpha<1$. The state trajectory of the closedloop system is shown in Figure 1. The switching signal $\sigma(k)$ is depicted in Figure 2. Figure 3 plots the evolution of $x(t) \alpha$, which implies that the corresponding closed-loop system is GCFTB with respect to $\left(\alpha, \beta, T_{f}, d, \sigma(k)\right)$, and the cost value $J^{*}=3.72$, which can be obtained by (35). 


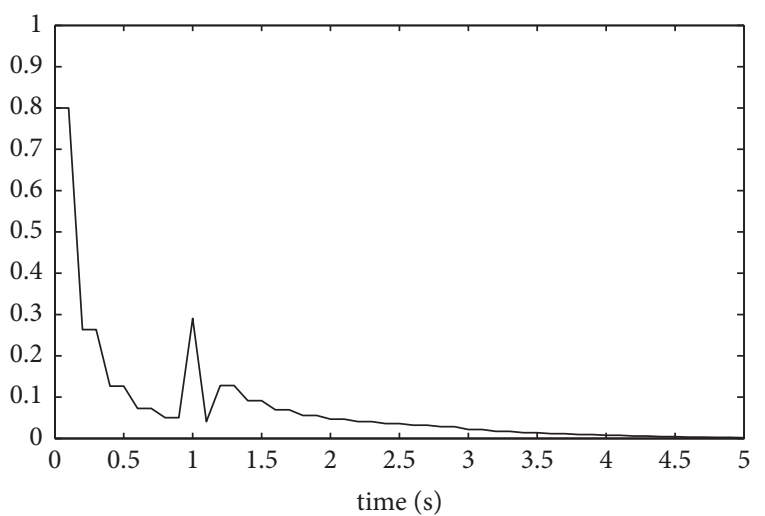

Figure 3: The evolution of $x^{T}(t) \alpha$ of system (1).

\section{Conclusions}

In this paper, we have considered the issue of guaranteed cost finite-time control for discrete-time positive impulsive switched systems. Based on the ADT approach, a guaranteed cost finite-time controller is constructed to guarantee that the closed-loop system is GCFTB. Finally, a numerical example is given to illustrate the effectiveness of the proposed method.

\section{Conflicts of Interest}

The authors declare that there are no conflicts of interest regarding the publication of this paper.

\section{Acknowledgments}

The authors are thankful for the support of the National Natural Science Foundation of China (under Grants nos. U1404610, 61473115, and 61374077) and Young Key Teachers Plan of Henan Province (2016GGJS-056).

\section{References}

[1] P. H. A. Ngoc, "Robust stability of positive linear systems under time-varying perturbations," Numerical Functional Analysis and Optimization, vol. 35, no. 6, pp. 739-751, 2014.

[2] J. Shen and S. Chen, "Stability and $L_{\infty}$-gain analysis for a class of nonlinear positive systems with mixed delays," International Journal of Robust and Nonlinear Control, vol. 27, no. 1, pp. 39-49, 2017.

[3] X. Zhao, Y. Yin, and J. Shen, "Reset stabilisation of positive linear systems," International Journal of Systems Science, vol. 47, no. 12, pp. 2773-2782, 2016.

[4] X. Zhao, X. Liu, S. Yin, and H. Li, "Improved results on stability of continuous-time switched positive linear systems," Automatica, vol. 50, no. 2, pp. 614-621, 2014.

[5] S. Liu and Z. Xiang, "Exponential $L_{1}$ output tracking control for positive switched linear systems with time-varying delays," Nonlinear Analysis: Hybrid Systems, vol. 11, pp. 118-128, 2014.

[6] G. Zong, H. Ren, and L. Hou, "Finite-time stability of interconnected impulsive switched systems," IET Control Theory \& Applications, vol. 10, no. 6, pp. 648-654, 2016.
[7] M. Xiang and Z. Xiang, "Robust fault detection for switched positive linear systems with time-varying delays," ISA Transactions $^{\circledR}$, vol. 53, no. 1, pp. 10-16, 2014.

[8] X. Zhao, L. Zhang, P. Shi, and M. Liu, "Stability of switched positive linear systems with average dwell time switching," Automatica, vol. 48, no. 6, pp. 1132-1137, 2012.

[9] J.-G. Dong, "Stability of switched positive nonlinear systems," International Journal of Robust and Nonlinear Control, vol. 26, no. 14, pp. 3118-3129, 2016.

[10] M. Xiang and Z. Xiang, "Stability, $L_{1}$-gain and control synthesis for positive switched systems with time-varying delay," Nonlinear Analysis: Hybrid Systems, vol. 9, pp. 9-17, 2013.

[11] T. Kaczorek, "Simple sufficient conditions for asymptotic stability of positive linear systems for any switchings," Bulletin of the Polish Academy of Sciences-Technical Sciences, vol. 61, no. 2, pp. 343-347, 2013.

[12] J. Zhang, Z. Han, and J. Huang, "Stabilization of discretetime positive switched systems," Circuits, Systems and Signal Processing, vol. 32, no. 3, pp. 1129-1145, 2013.

[13] E. Fornasini and M. E. Valcher, "Stability and stabilizability criteria for discrete-time positive switched systems," Institute of Electrical and Electronics Engineers Transactions on Automatic Control, vol. 57, no. 5, pp. 1208-1221, 2012.

[14] M. A. Rami, F. Tadeo, and A. Benzaouia, "Control of constrained positive discrete systems," in Proceedings of the American Control Conference (ACC '07), pp. 5851-5856, New York, NY, USA, July 2007.

[15] D. Peter, "Short time stability in linear time-varying systems," IRE International Convention Record, vol. 4, pp. 83-87, 1961.

[16] G. Chen and Y. Yang, "Finite-time stability of switched positive linear systems," International Journal of Robust and Nonlinear Control, vol. 24, no. 1, pp. 179-190, 2014.

[17] M. Xiang and Z. Xiang, "Finite-time $L_{1}$ control for positive switched linear systems with time-varying delay," Communications in Nonlinear Science and Numerical Simulation, vol. 18, no. 11, pp. 3158-3166, 2013.

[18] J. Zhang, X. Zhao, and Y. Chen, "Finite-time stability and stabilization of fractional order positive switched systems," Circuits, Systems and Signal Processing, vol. 35, no. 7, pp. 24502470, 2016.

[19] L. Liu, X. Cao, Z. Fu, and S. Song, "Input-output finite-time control of positive switched systems with time-varying and distributed delays," Journal of Control Science and Engineering, vol. 2017, Article ID 4896764, 9 pages, 2017.

[20] J. Liu, J. Lian, and Y. Zhuang, "Output feedback $L_{1}$ finite-time control of switched positive delayed systems with MDADT," Nonlinear Analysis: Hybrid Systems, vol. 15, pp. 11-22, 2015.

[21] J. Zhang, Z. Han, and H. Wu, "Robust finite-time stability and stabilisation of switched positive systems," IET Control Theory \& Applications, vol. 8, no. 1, pp. 67-75, 2014.

[22] T. Liu, B. Wu, L. Liu, and Y.-E. Wang, "Asynchronously finitetime control of discrete impulsive switched positive time-delay systems," Journal of The Franklin Institute, vol. 352, no. 10, pp. 4503-4514, 2015.

[23] G. Zong, X. Wang, and H. Zhao, "Robust finite-time guaranteed cost control for impulsive switched systems with time-varying delay," International Journal of Control, Automation, and Systems, vol. 15, no. 1, pp. 113-121, 2017.

[24] L. Liu, M. Cheng, and M. Xu, " $H_{\infty}$ guaranteed cost finite-time control for stochastic differential inclusion systems," in Proceedings of the 2015 IEEE International Conference on Information and Automation, pp. 1447-1451, China, August 2015. 
[25] D. Yang and K.-Y. Cai, "Finite-time reliable guaranteed cost fuzzy control for discrete-time nonlinear systems," International Journal of Systems Science, vol. 42, no. 1, pp. 121-128, 2011.

[26] C. Liu and W.-H. Chen, "Guaranteed cost control of linear uncertain discrete-time impulsive systems," Transactions of the Institute of Measurement and Control, vol. 37, no. 1, pp. 33-39, 2015.

[27] J. Huang, Z. H. Guan, and Z. D. Wang, "Guaranteed cost control for uncertain discrete-time impulsive systems," Journal of Huazhong University of Science and Technology, vol. 34, no. 2, pp. 1238-1242, 2006.

[28] L. Liu, X. Cao, Z. Fu, and S. Song, "Guaranteed cost finite-time control of fractional-order positive switched systems," Advances in Mathematical Physics, vol. 2017, Article ID 2695894, 11 pages, 2017.

[29] X. Cao, L. Liu, Z. Fu, X. Song, and S. Song, "Guaranteed cost finite-time control for positive switched linear systems with time-varying delays," Journal of Control Science and Engineering, vol. 2017, Article ID 7051658, 10 pages, 2017.

[30] H. Xing, L. Liu, X. Cao, Z. Fu, and S. Song, "Guaranteed cost finite-time control of positive switched nonlinear systems with D-perturbation," Open Mathematics, vol. 15, pp. 1635-1648, 2017. 


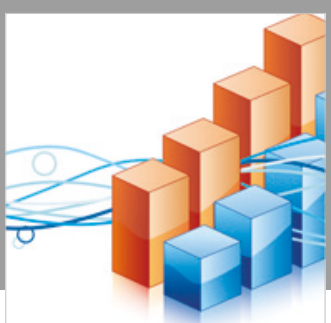

Advances in

Operations Research

\section{-n-m}
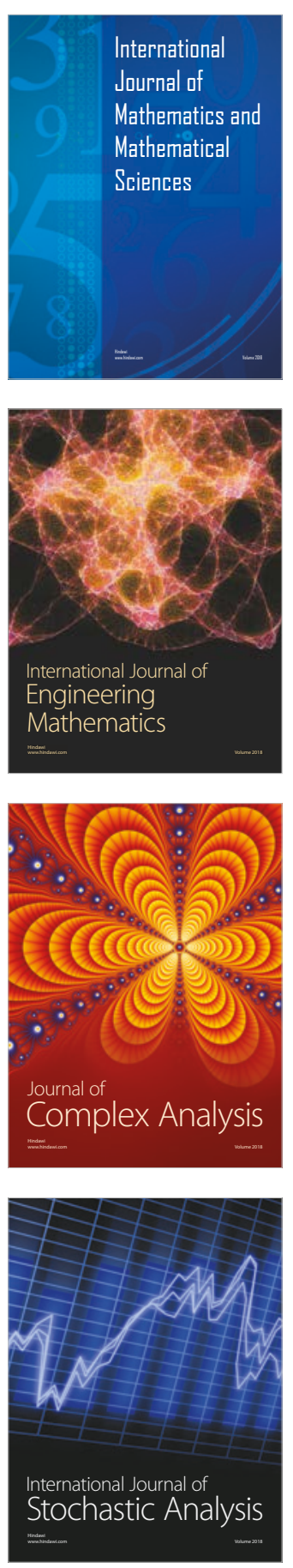
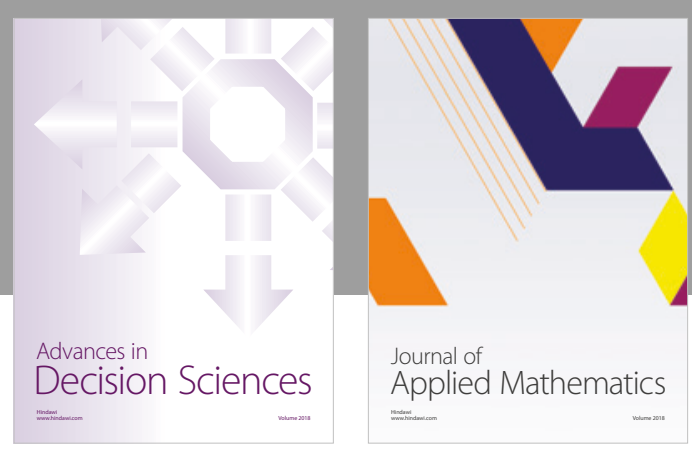

Journal of

Applied Mathematics
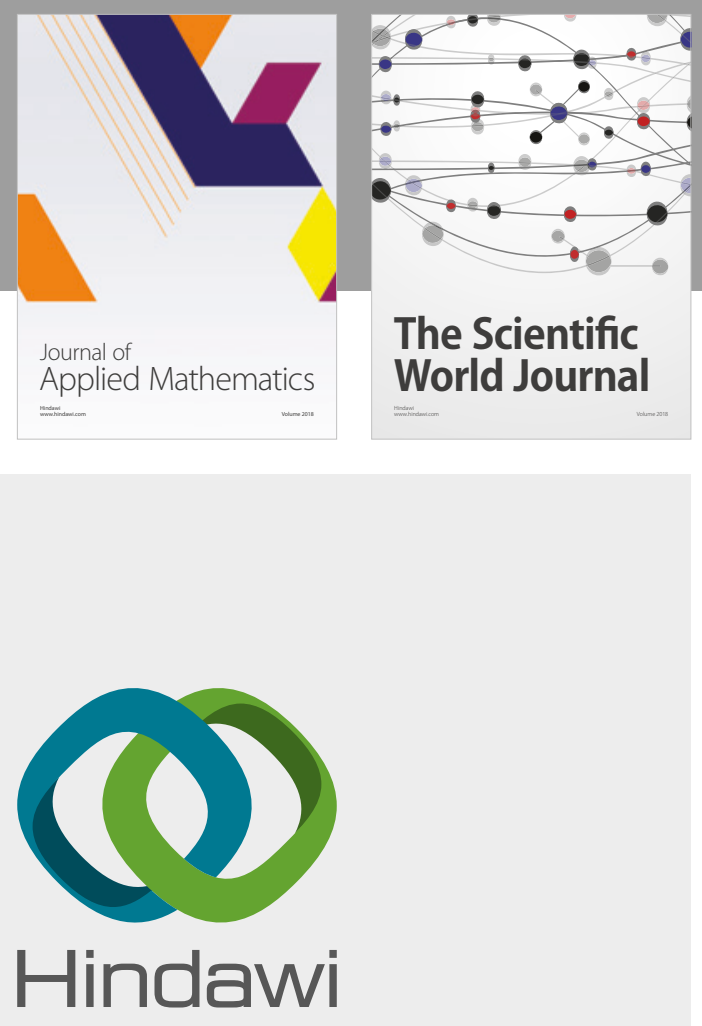

Submit your manuscripts at

www.hindawi.com

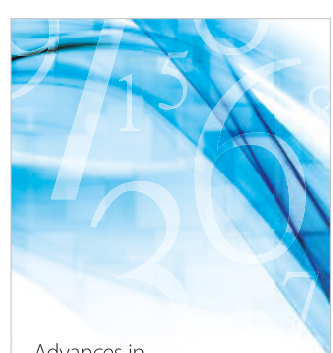

Advances in
Numerical Analysis
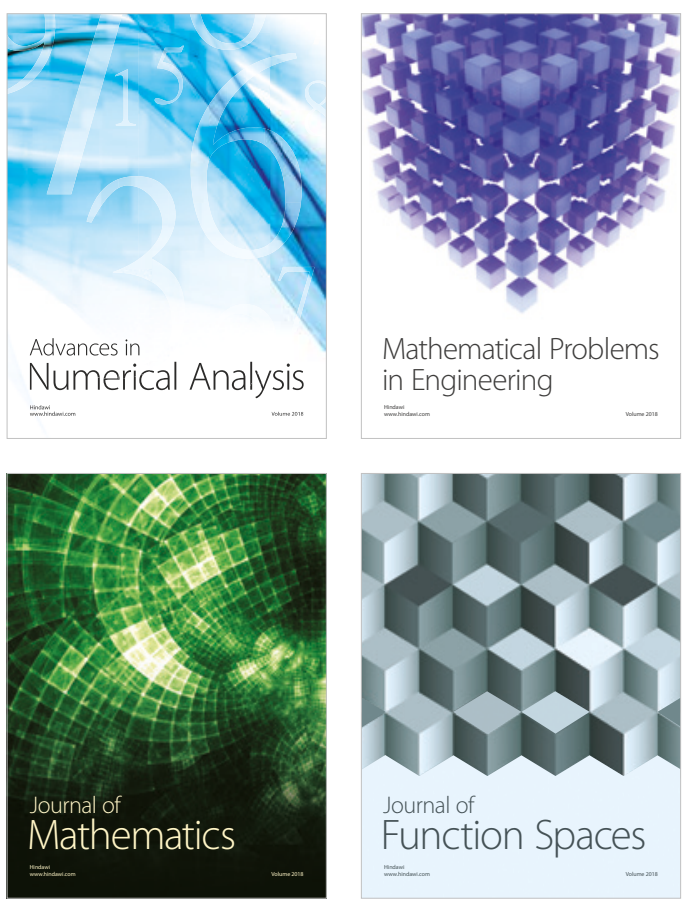

Mathematical Problems in Engineering

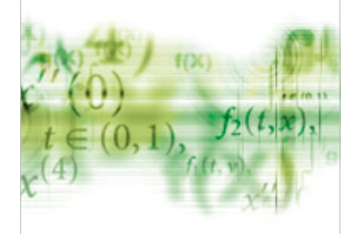

International Journal of

Differential Equations

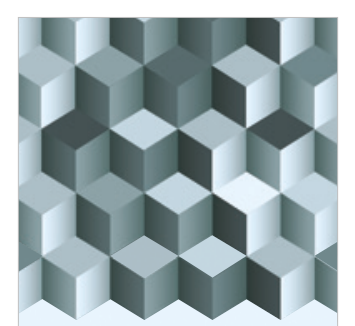

Journal of

Function Spaces
The Scientific

World Journal

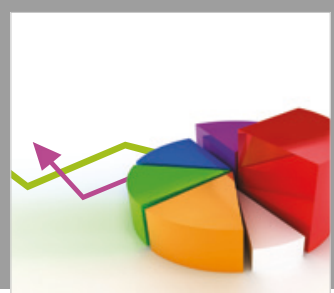

Journal of

Probability and Statistics
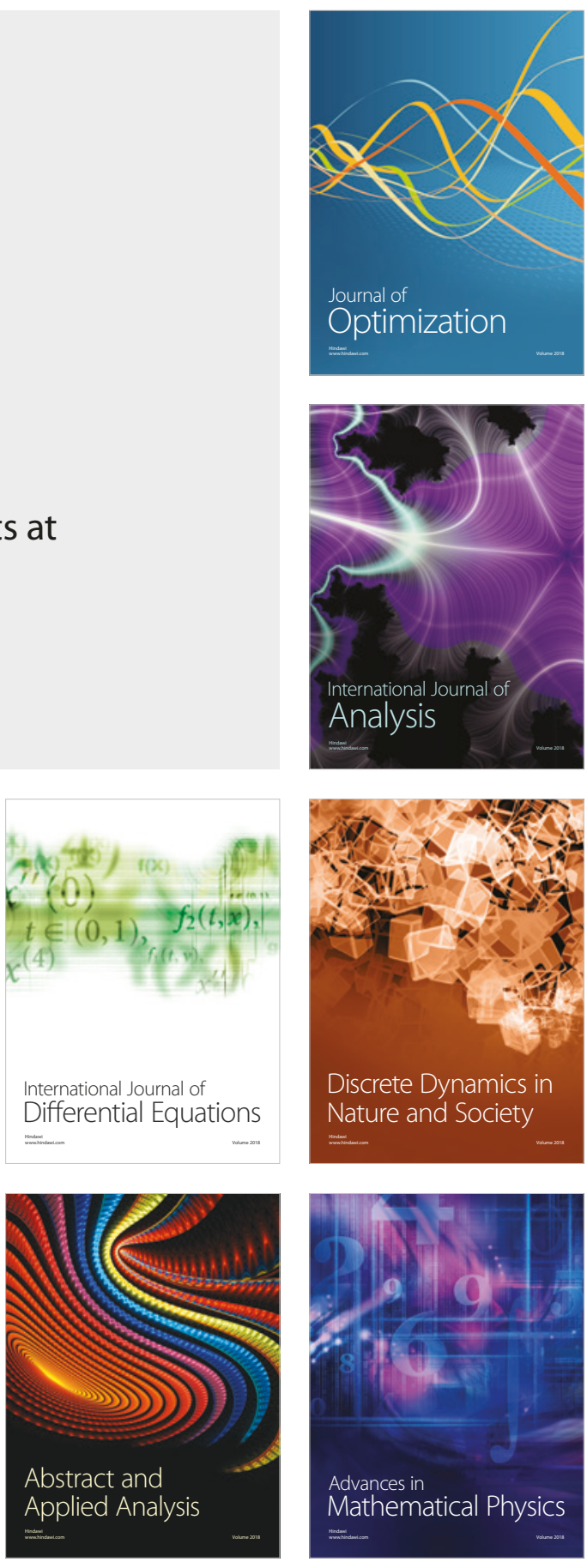